\title{
Treatments aim to topple papillomavirus before cancer begins
}

Between the recently approved prophylactic vaccines against the human papillomavirus (HPV), and the ease with which surgery can treat most cervical cancers, therapeutic treatments for the virus have been low on most clinicians' priority lists. But with increasing incidence of other HPV-associated cancers that are more difficult to treat surgically-for example, oral cancer-a new generation of therapies aimed at HPV-induced precancerous lesions and warts could be on the horizon.

Traditionally, therapies that have tackled HPV have done so by broadly boosting the immune system. But new approaches under investigation take a more specific tack. For example, rather than enhancing a general immune response, Eberhard Amtmann, a virologist at the German Cancer Research Center in Heidlberg, is trying to target a specific host-expressed enzyme that HPV relies on to replicate in human cells.

Current prophylactic vaccines, such as Gardasil and Cervarix, and therapeutic vaccines tend to combat only two of the most cancer-causing strains of HPV, leaving people susceptible to many other strains that are responsible for around $30 \%$ of cervical cancers. Targeting the host enzyme, however, might work against all viral types.

"When you inhibit this cellular enzyme, you can treat all kinds of human papillomavirus infections," says Amtmann, who presented in vitro results with a small-molecule drug that inhibits a key signal transduction enzyme at the International Congress on Infectious Diseases in Miami in March. Further experiments, which will be presented this month at the Society for Investigative Dermatology meeting in Atlanta, showed that the drug inhibited tumor growth in mice infected with a high-risk strain of HPV.

\section{Options for women}

"The ability to treat a viral infection that at the moment is left untreated and can lead to cancer is a major breakthrough for many women," says Nicholas Benedict, chief executive of the Swiss biotech Lumavita, which is co-developing the compound. Phase 1 clinical trials combining the drug with a newly developed gel that is well tolerated in the genital tract are planned for later this year, he says.

"At the moment, patients who are HPVpositive have no treatment options-just more follow-ups and looking for lesions," Benedict adds. "This would fit in earlier in the treatment cascade."
Meanwhile, Thomas Broker and Louise Chow, a husband-and-wife team at the University of Alabama-Birmingham, are hoping to directly target the virus itself. Last year, they developed one of the first efficient systems for culturing the virus in human cells in vitro (Genes Dev. 23, 181-194, 2009). And now, using this cell culture system, the researchers have blocked two different parts of the mitogen-activated protein (MAP) kinase signaling pathway to prevent viral replication.

"We're absolutely convinced [that targeting these pathways] will lead to drugs," says Broker, president of the International Papillomavirus Society, of his unpublished results. And, like Amtmann's approach, preventing viral replication should be effective for all strains of HPV, Chow adds.

\section{Boosting therapeutic vaccines}

Researchers have developed a number of therapeutic vaccines to tackle such lesions before they grow into tumors, but these products, too, have shown mixed efficacy. Now, some experts have suggested that combining these therapeutic vaccines with a localized remedy might work.

The most common noninvasive remedy for genital warts and some skin cancers is a topical cream called imiquimod, which stimulates the immune system through the Toll-like receptor pathway to fight HPV. But imiquimod doesn't work for all patients, and it has some nasty side effects, such as irritation of mucosal surfaces. Moreover, the cream is only effective at the site of application, which necessitates that people spot all warts and potentially cancerous lesions.

Ian Frazer, an immunologist at the University of Queensland in Brisbane, Australia, who helped develop the preventative HPV vaccine Gardasil, thinks that combining these two approaches is the way forward. "The combination of vaccine and local immunotherapy probably has the best prospect of getting something to work for persistent HPV infection," he says.

This was the strategy adopted by Peter Stern and his colleagues at the University of Manchester, UK, who conducted a phase 2 trial in which they treated 19 women with $\mathrm{HPV}$-associated vulval precancerous lesions with imiquimod followed by a therapeutic vaccine. Reporting in March, the researchers found that the double-whammy approach altered the local balance between effector $\mathrm{T}$ cells, which help fight infection, and regulatory

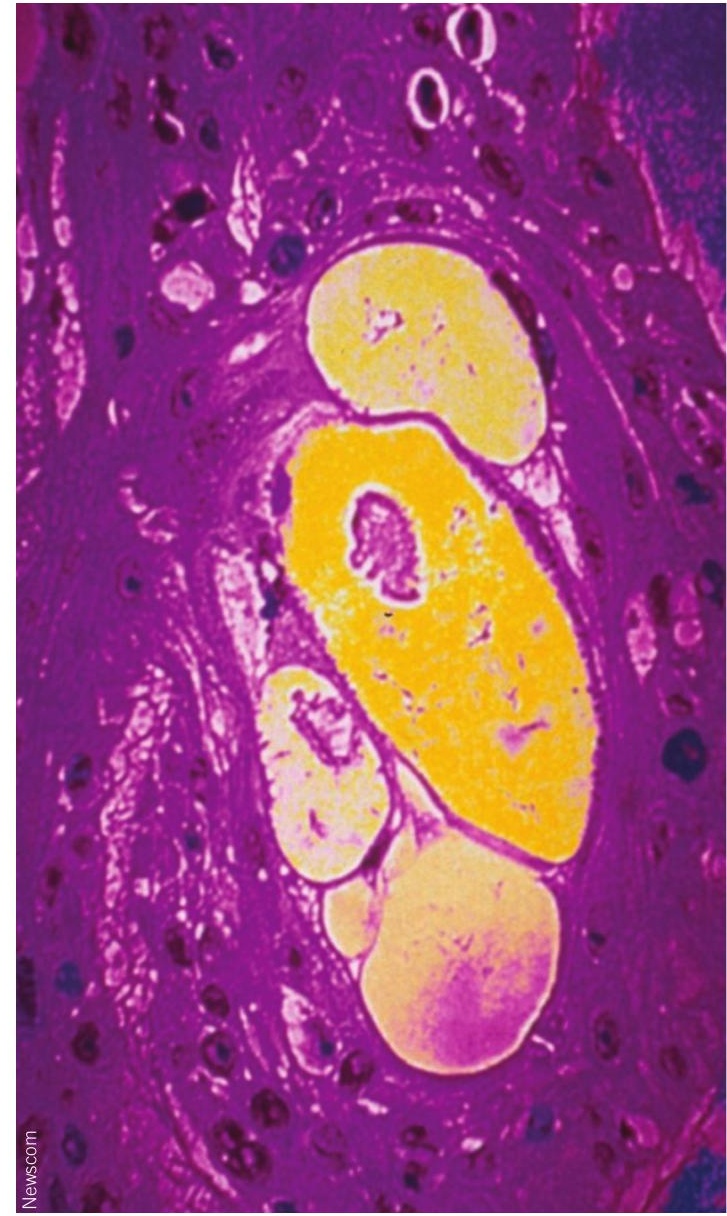

Cervix and protect: Potential HPV drugs.

T cells, which suppress immune activity, so that the majority of women had no symptoms one year after receiving the treatment $(B r . J$. Cancer 102, 1129-1136, 2010).

"There's a balance between the good guys and the bad guys," says Stern. This treatment "changes the local immune response so as to allow the immune system to work better." But, like treatment with either the cream or the vaccine on its own, many women did not respond to the dual therapy at all.

Despite the intrinsic challenges to treating active HPV infections, Diane Harper, director of the gynecologic cancer prevention research group at the University of Missouri-Kansas City School of Medicine, applauds efforts to find noninvasive ways to eliminate these infections. "If we can develop a therapeutic agent for HPV that can help speed the immune clearance, we would have a wonderful adjuvant to all the other therapies that we currently can offer women," she says.

Elie Dolgin, New York 